\title{
Erratum: Dark Matter from Exponential Growth [Phys. Rev. Lett. 127, 191802 (2021)]
}

\author{
Torsten Bringmann $\odot$, Paul Frederik Depta, Marco Hufnagel, Joshua T. Ruderman, and Kai Schmidt-Hoberg
}

(Received 12 January 2022; published 10 February 2022)

DOI: 10.1103/PhysRevLett.128.069901

In our Letter, we provide an example realization of dark matter (DM) production via exponential growth, where the heat bath particle $\psi$ is coupled to the standard model (SM) via the Higgs portal, $\lambda_{h \psi}|H|^{2} \psi^{2} / 2$. The DM particle $\chi$ is initially produced by freeze-in via $\psi \psi \rightarrow \chi \chi$, induced by the term $\lambda_{\mathrm{fi}} \psi^{2} \chi^{2} / 4$, until the transformation process $\chi \psi \rightarrow \chi \chi$, as induced by a term $\lambda_{\text {tr }} \psi \chi^{3} / 6$, takes over and leads to exponential growth. By connecting one outgoing $\psi$ of the Higgs-portal vertex to a transformation vertex, cf. Fig. 6, one, however, also obtains a freeze-in contribution via SM SM $\rightarrow \psi \chi \chi \chi$ that we missed in our analysis. By calculating the exact cross-section for this process using Feynrules [1] and MADGRAPH5 [2], we find that this additional process produces more DM than $\psi \psi \rightarrow \chi \chi$ if $\lambda_{\mathrm{fi}} \lesssim \lambda_{h \psi} \lambda_{\mathrm{tr}} /\left[3(4 \pi)^{2}\right]$, where we explicitly find an enhancement of $\sim 3$ compared to the factor $(4 \pi)^{2}$ naïvely resulting from phase-space suppression. This condition is satisfied within the entire parameter region shown in Fig. 5 of this Letter, implying that we underestimated the initial abundance before the onset of exponential growth, leading to an overproduction of DM in the entire parameter region. In particular, the possibility of exponential DM production for large $m_{\psi}>1 \mathrm{TeV}$ in the Higgs-portal example is excluded.

To allow for exponential growth in the Higgs-portal example, we thus need a smaller coupling $\lambda_{h \psi}$, without $\psi$ becoming the dominant DM component. In order not to violate limits from invisible Higgs decays [3] or direct detection with Xenon1T [4], this only leaves the region of resonant $\psi$ annihilations, $m_{\psi} \sim m_{h} / 2$ [5]. We show an explicit example for $m_{\psi}=m_{h} / 2$ in Fig. 7. The relic density of $\psi$ is set by freeze-out, where we took into account the effect of early kinetic decoupling as implemented in DarkSUSY [6] and we adjust the coupling to $\lambda_{h \psi}=3.9 \times 10^{-4}$ such that $\psi$ accounts for $20 \%$ of DM. We then adjust $\lambda_{\mathrm{fi}}$ for every $m_{\chi}$ and $\lambda_{\text {tr }}$ to produce $80 \%$ of the total DM abundance in $\chi$. The dominant production mechanisms are indicated by green and blue regions for freeze-in and exponential growth, respectively. In the light blue region, 2-to-4 freeze-in via the Higgs portal, neglected in our original calculation, dominates freeze-in production over $\psi \psi \rightarrow \chi \chi$, generally leading to DM overproduction as discussed above.

Because of the decreased value of $\lambda_{h \psi}$, the cosmic-microwave-background (CMB) signatures from $\psi$ decay disappear for $m_{\chi}<m_{\psi}$. Hence, we focus on the region $m_{\chi}>m_{\psi}$, where $\chi$ decays into $\psi$ and SM final states, predominantly by two-loop diagrams involving one freeze-in and one transmission vertex as well as the Higgs portal to a (potentially off-shell) Higgs boson decaying into SM final states. (Possible three-loop diagrams with three transmission vertices are suppressed as $\lambda_{\text {tr }}$ cannot get large enough without reaching the light blue region.) We roughly estimate a decay width of

$$
\Gamma_{\chi} \simeq \frac{\lambda_{h \psi}^{2} v^{2}}{16 \pi m_{\chi}^{3}} \frac{\lambda_{\mathrm{tr}}^{2} \lambda_{\mathrm{fi}}^{2}}{(4 \pi)^{8}} \int_{0}^{\left(m_{\chi}-m_{\psi}\right)^{2}} \frac{d q^{2}}{2 \pi} 2 q \Gamma_{h}(q) \times \frac{\sqrt{\left[m_{\chi}^{2}-\left(m_{\psi}+q\right)^{2}\right]\left[m_{\chi}^{2}-\left(m_{\psi}-q\right)^{2}\right]}}{\left(q^{2}-m_{h}^{2}\right)^{2}+m_{h}^{2} \Gamma_{h}^{2}\left(m_{h}\right)},
$$

FIG. 6. Feynman diagram for 2-to-4 freeze-in via the Higgs portal.

Published by the American Physical Society under the terms of the Creative Commons Attribution 4.0 International license. Further distribution of this work must maintain attribution to the author(s) and the published articles title, journal citation, and DOI. 


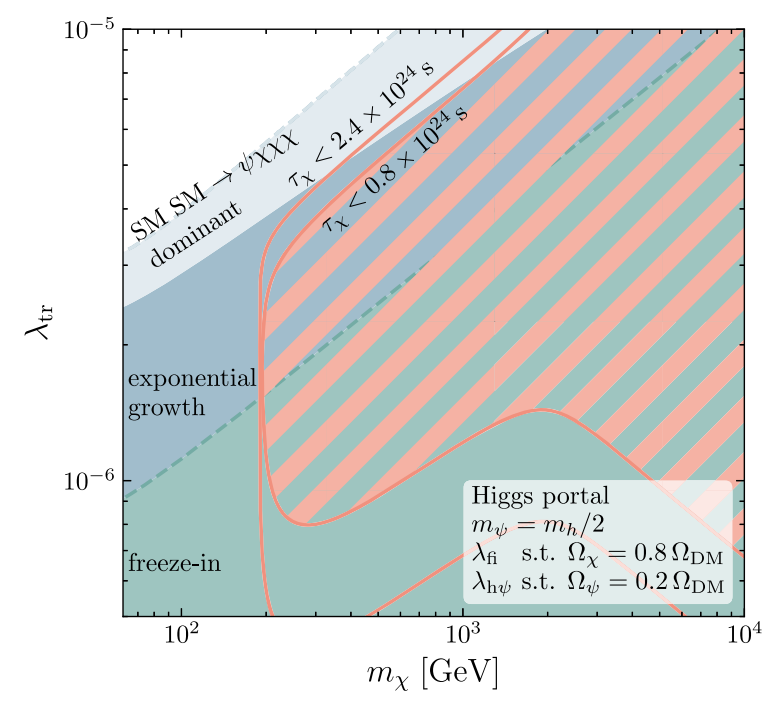

FIG. 7. Phenomenological consequences of a specific example realization of exponential production, where $\psi$ is coupled to the SM via the Higgs portal in the regime of resonant annihilations $\left(m_{\psi}=m_{h} / 2\right)$. We fix $\lambda_{h \psi}=3.9 \times 10^{-4}$, giving $20 \%$ of DM in $\psi$, and choose $\lambda_{\mathrm{fi}}$ for each $m_{\chi}$ and $\lambda_{\text {tr }}$ such that $\chi$ provides $80 \%$ of the observed DM abundance. Green and blue regions indicate which production mechanism dominates the $\chi$ abundance. Red lines (and the hatched area) show where CMB signatures can naturally be expected. Within the light blue region, freeze-in via $h h \rightarrow \psi \chi \chi \chi$ dominates the initial $\chi$ abundance over $\chi \chi \rightarrow \psi \psi$, thus generically leading to overproduction of DM.

where $v \approx 246 \mathrm{GeV}$ is the Higgs vacuum expectation value, and $\Gamma_{h}(q)$ is the off-shell decay width of a Higgs boson with invariant mass $q$ (which we evaluate using HDECAY [7]). This decay can alter the CMB power spectrum, constrained to a lifetime $\tau_{\chi}=1 / \Gamma_{\chi} \gtrsim 0.8 \times 10^{24} \mathrm{~s}$ for $80 \%$ of DM in $\chi$ [8]. CMB-S4 is expected to strengthen this constraint by a factor of $\sim 3$ [9]. We show where the naïve expectation of the lifetime is in conflict with these constraints or may be tested by CMB-S4 with the hatched area and red lines in Fig. 7. For $m_{\chi}<m_{\psi}+m_{h}=(3 / 2) m_{h}$, the Higgs can only be produced off shell, resulting in a significant suppression of the decay width and hence the absence of CMB limits in this parameter range.

J. T. R. thanks the participants of the workshop "New Physics from the Sky" at the Galileo Galilei Institute for helpful feedback. This work is supported by the Deutsche Forschungsgemeinschaft under Germany's Excellence StrategyEXC 2121 "Quantum Universe"-390833306, the F.R.S.-FNRS under the Excellence of Science (EoS) project No. 30820817-be.h "The H boson gateway to physics beyond the standard model," and the National Science Foundation under Grant No. NSF PHY-1748958. J. T. R. is further supported by the NSF CAREER Grant No. PHY1554858, NSF Grant No. PHY-1915409, an award from the Alexander von Humboldt Foundation, and the European Research Council (ERC) under the EU Horizon 2020 Programme (ERC-CoG-2015-Proposal No. 682676 LDMThExp).

[1] A. Alloul, N. D. Christensen, C. Degrande, C. Duhr, and B. Fuks, Feynrules2.0-A complete toolbox for tree-level phenomenology, Comput. Phys. Commun. 185, 2250 (2014).

[2] J. Alwall, M. Herquet, F. Maltoni, O. Mattelaer, and T. Stelzer, MADGRAPH5: Going beyond, J. High Energy Phys. 06 (2011) 128.

[3] The ATLAS Collaboration, Combination of searches for invisible Higgs boson decays with the ATLAS experiment, Report No. ATLAS-CONF-2020-052, 2020.

[4] E. Aprile et al. (XENON Collaboration), Dark Matter Search Results from a One Ton-Year Exposure of XENON1T, Phys. Rev. Lett. 121, 111302 (2018).

[5] P. Athron et al. (GAMBIT Collaboration), Status of the scalar singlet dark matter model, Eur. Phys. J. C 77, 568 (2017).

[6] T. Binder, T. Bringmann, M. Gustafsson, and A. Hryczuk, DRAKE: Dark matter relic abundance beyond kinetic equilibrium, Eur. Phys. J. C 81, 577 (2021).

[7] A. Djouadi, J. Kalinowski, M. Muehlleitner, and M. Spira, HDECAY: Twenty ++ years after, Comput. Phys. Commun. 238, 214 (2019).

[8] T. R. Slatyer and C.-L. Wu, General constraints on dark matter decay from the cosmic microwave background, Phys. Rev. D 95, 023010 (2017).

[9] K. N. Abazajian et al. (CMB-S4 Collaboration), CMB-S4 Science Book, first ed., arXiv:1610.02743. 